\title{
A Visualization Analysis of China's Leisure Sports Research
}

\author{
Sun Ming, Zeng Ji \\ School of Physical Education \\ Hubei University \\ Wuhan, China \\ Corresponding Author: Zeng Ji, 1337266477@qq.com
}

\author{
Hu Tian \\ Center for Studies of Education and Psychology of \\ Ethnic Minorities In Southwest China \\ Southwest University \\ Chongqing, China \\ 454816870@qq.com
}

\begin{abstract}
The social economy's continual development has produced gradual increases in disposable income, which have enriched leisure activities. In order to engage progress in the research of leisure sports in China over the past 20 years and contribute a new angle and direction to the research of leisure sports in China, this paper summarizes the internal and external characteristics of the country's leisure sports research. The data visualization software CiteSpace5.0 is applied to provide a macro analysis of the development of China's leisure sports research over the past 20 years. This method has not been applied to research of this kind before, and this reiterates the innovative character of this paper. It achieves this by extracting the 1998-2017 leisure sports literature from the CSSCI database before applying bibliometric Visual Analysis Software CiteSpace 5.0 to this material. It finds that the momentum of the development of the county's leisure sports is positive, and observes the initial establishment of a cooperation network. However, it also highlights a number of problems, which include the insufficient depth of disciplinary research, the lack of interdisciplinary research results (as shown by the adoption of a single research paradigm) and the neglect of frontier issues.
\end{abstract}

DOI reference number: 10.18293/DMSVIVA20-005
Keywords-Chinese Leisure Sports; Leisure Research Hotspots; Leisure Sports Research; Visualization Analysis

\section{INTRODUCTION}

China's leisure sports research mainly focuses on the history, development status and problems encountered by leisure sports research. Although this research had a late start (Kong Chuihui, 2009), its development has been rapid and it has provided enriching perspectives and generated methods that can be applied (Xiaoyu and Hai, 2016).

But there are a number of general problems in the study of China's leisure sports that need to be resolved. These are as follows: 1) The definition of the leisure sports concept is ambiguous and is not sufficiently comprehensive $(\mathrm{Wu}$ Jiangang, 2003; Li Xiangru, 2015); (2) Leisure sports research has limited depth and its results are not sufficiently influential (Xiaoyu and Hai, 2016); (3) Most of the research has an international focus, and does not sufficiently refer to China (Xiaoyu and Hai, 2016); (4) a single research method tends to be adopted and cooperative and interdisciplinary research is 
limited; (ibid) (5) research groups are not sufficiently diverse and do not sufficiently acknowledge engagement in leisure sports activities across different social classes (Jiangang, 2003; Chuihui, 2009).

There are grounds for believing that the development of leisure sports will make an important contribution to the rapid transformation of Chinese society and its future modernization. China therefore provides an ideal opportunity to study the relationship between leisure sports research and the stage of national development. Here it should be remembered that most of the research samples of leisure sports have been taken from Western contexts. The study of the development of China's leisure sports research over the past 20 years will provide insight into its future direction.

This paper will explore dynamic changes in China's leisure sports hotspots during the period 1998-2018; it does so with the intention of identifying key characteristics of China's leisure sports research, predicting future research trends and highlighting problems that can be engaged by Chinese researchers. Although development trends in China's leisure sports research have been extensively engaged (Jiangang, 2003; Limin, 2007; Xiaoming, 2008; Chuihui, 2009; Xin, 2012; Xiaoyu, 2016; Xiuyu, 2016), there are still research shortcomings that need to be directly addressed. This paper seeks to contribute to this process by providing an overview of all literature related to leisure sports topics that were published by CSSCI journals and incorporated into the China Knowledge Network (CNKI) during the period 1998-2018. It will apply Citespace with the intention of gaining insight into the literature's features, strengths and deficiencies, along with its dynamic development.

\section{LITERATURE REVIEW}

China is the world's second largest economy. In 2018, its gross domestic product (GDP) reached $\$ 90.03$ trillion (USD) and its population expanded to 1.395 billion, accounting for about one-fifth of the world's population. (World Bank, 2018) Since the 1980s, China's annual economic growth rate has, on average, exceeded 9 percent, which far surpasses the annual growth rate of 2.3 percent recorded for developed economies during the same period. (World Bank, 2011). Rapid economic development has greatly improved Chinese living standards ( $\mathrm{Li}$ et al., 2012) and residents of mainland China now have the time and financial resources to participate in leisure activities. (Liang \& Walker, 2011).

In recent times, China's booming economy and overall national strength has created huge dividends for its citizens. Personal income has also increased as a result of more efficient resource allocation and more incentives for private investment (Easterlin et al., 2012). During this transition period, both the leisure time (Yin, 2005) and leisure consumption of Chinese residents have increased significantly (Zhai \& Xiao, 2004).

\section{A. The conceptualization of 'leisure sports'}

Chinese scholars have not yet reached an agreement on what 'leisure sports' are, and this has interrupted research into conceptualization. The most renowned experts in this area are Tian Hui, Ma Huizhen, Zhou Aiguang, Lu Feng and Xiao Huanqi. Ma Huizhen (2008) and Zhou Aiguang (2009). They define 'leisure sports' in similar terms, and observe they are a sports activity engaged in for leisure, which relieves stress, eliminates fatigue and benefits body and mind. Tian Hui (2006), Xiao Huanqi (2010) and Yu Kehong (2003) define 
them as sports activities that are engaged in during leisure time, which assist economic, environmental, personal and social development. Lu Feng (2004) suggests they are a collective term for various sports activities engaged under relatively free conditions.

The classification and definition of 'leisure sports' by scholars shows that China lacks metacognition of this concept . It is part of a foreign vocabulary, and has no Chinese counterpart. The word needs to be traced back to its root in order to explore its original meaning.

\section{B. Leisure Sports and individual and collective development}

A substantial amount of research has engaged with the developmental contribution of leisure sports. Since the beginning of the $21 \mathrm{st}$ century, population and economic aggregates have both increased and the individual and collective influence of leisure sports has attracted growing academic interest. Lu Feng (2004) suggests that the development of leisure sports has personal, developmental, social, social fashion (communication), social group (organization) and social symbolic functions. Zhang Rui (2014) observes that leisure sports do not just enhance physical health but also improve mental health, promote social interaction, create a relaxed and comfortable environment and contribute to an improved quality of life.

It is only by understanding and promoting leisure sports, in addition to grasping their essential purpose, that it will be possible to promote a healthier body and mind. Lu Gaofeng (2014) suggests that leisure sports provide a basis for various capital conversions. By engaging in leisure sports, higher social classes can accumulate cultural capital, expand social capital, and maintain or develop physical capital by investing in economic capital. Lower social classes can also transform physical capital into economic capital by engaging in leisure sports or can instead transform it into a form of cultural or social capital.

Qiu Yajun (2014) finds that women who do not participate in leisure sports are mainly held back by their own limitations and structural constraints. Female participants in recreational sports activities frequently encounter perceptual and experience limitations. Xiong Huan (2014) suggests that sport dissolves individual and micro-level limitations of women's leisure, and therefore promotes their freedom of choice and empowerment - this, however, is conditional on a more equal, free and reasonable social system. Only when the cultural environment is present will it be possible to effectively achieve this.

Jinyin Day (2015) finds that the leisure sports activit ies of Shanghai residents are characterized by activity spa ce circle, activity demand differentiation, wide-area charac teristics of travel space and the regularization of activity time. Wu Xiaoyang's (2015) research of the literature de monstrates that leisure sports are one way through which rural citizens integrate into an urban environment. The num ber of people involved in leisure sports therefore provides $\mathrm{i}$ nsight into the extent of urbanization.

Ye Xin (2015) proposes that gender order is the most important part of women's leisure sports behavior, and suggests it establishes a basis for consciousness while putting in place a material foundation that underpins women's leisure sports behavior. Yan Ke (2016) suggests that the healthy development of leisure sports relies on a benign interaction between individual self-development and social norms. This 
entails that the psychology and behavior of social individuals will produce their active participation in leisure sports and will encourage their use of social laws, regulations, norms and ethics that control, regulate and stimulate society. Guo Xiujin (2016) observes that the best popular leisure sports involve nature and civilization, and she therefore presents leisure sports as a kind of 'green living style' that helps to make the world a more harmonious place. Yan Ke (2017) notes that individuals make the nation's leisure and sports life possible, and observes the development of national leisure sports promotes an accelerated individualization. Fashion and market tendencies are the social characteristics that correspond to the development of national leisure sports in an individualized era.

Chen Dexu (2017) notes how leisure sports have pro moted material development by stimulating sports consum ption and economic growth, strengthening social governan ce, enhancing the stability of political civilization, improvi ng the quality of the population and promoting the health y development of utility. Sun Fenglin (2018) applies the theory of the ecological food chain network to a case stu dy, and observes that the park can meet the multi-level a nd multi-type leisure needs of the elderly. Wang Min (20 18) suggests that leisure sports are the most active, effect ive and economical way to deal with an aging society. T hey can enhance the physical condition, mental health an d life satisfaction of the elderly, while reducing their risk of illness. They can alleviate decreased participation in $\mathrm{t}$ he labour market caused by an aging society, and can als o reduce healthcare expenditures and the social burden.

Li Hui (2018) observes that women participate in leisure sport activities, and notes that this does not just challenge the traditional gender order, but also provides a new approach to the construction of this order in the new era. It also enables a healthy China to be constructed along both vertical and horizontal axes. In the first sense, the participation of women in leisure sports activities are based on a challenge to the traditional gender order, the realization of gender role self-identification, an empowered gender freedom, the expansion of gender space and the promotion of women's individuality on terms that encompass the whole life-cycle.

Chinese scholars have a lot of empirical research on the development of human and social development in leisure sports and there are few theoretical innovations. The research results mainly reflect the subsidiary meaning of leisure sports, and the interpretation of its essential functions is less, and further research is needed.

\section{Research into leisure sports majors}

Since the start of the century, China's comprehensive national strength has continually grown, along with related sport activities. The concept of 'leisure sports' has become increasingly widely accepted. Colleges and universities have also established leisure sports-related majors that help to meet growing demand for professional leisure sports in China. Because the major is newly developing, it is not amateur enou gh.The construction of China's leisure sports major has gradually become a research 'hot spot'.

Peng Guoqiang (2014) compares the construction of leisure sports majors in American colleges and universities, and proposes that China's leisure sports majors should meet the needs of the social market, cultivate composite talents that combine sports and leisure, broaden the coverage of leisure sports courses and reflect the curriculum. 
The dynamic development of the system, the reform and construction of the leisure sports curriculum and the professional setting reflect diversified social needs, the establishment of a third-party specialized agency and the need for a leisure sports professional audit system that is adapted to China. Wang Xiaoyun (2017) observes that current talent training in the construction of leisure sports is not closely integrated with social requirements, neglects the improvement of comprehensive quality, and also gives insufficient attention to professional practice and the low standardization of training programs. He suggests the country should be market-oriented, should analyze demand for leisure sports professional ability, should clarify the training specifications of leisure sports professionals and should build a competency-oriented and standard curriculum system that helps to achieve the overall optimization of leisure sports professionals. Xu Dapeng (2017) conducts a comparative study of leisure sports majors and social sports majors in the Capital Institute of Physical Education, which concludes that the professional setting of leisure sports should focus on innovation and entrepreneurship education, increase the specific sport choices that are available and also inspire students' innovative spirit.

Due to the lack of a correct understanding of the term "leisure sports", Chinese scholars have limited research on leisure sports majors, and the relevant results are not rich, and there is no research result that reflects the connotation of leisure sports.

D. The industrialization of leisure sports

The sports and leisure industry has become indispensable to China's national economic development and its construction of a harmonious society. The question of how to align China's leisure sports industry with national conditions has come to preoccupy many scholars. Luo Lin (2006) draws on the perspective of cultural chemistry to put forward three principles for the development of the leisure sports industry, specifically an orientation towards people, the accommodation of multicultural values and the development of a national traditional sports culture and valued leisure industry. Wang Xianliang (2015) finds that China's leisure sports industry is characterized by consumption and production simultaneity, industrial integration and sports characteristics. The development of the leisure sports industry should clarify the industrial value chain, optimize the value chain layout and give regional advantages in order to optimize regional layout. Ye Xiaoyu (2016) finds the research of China's leisure sports industry is developing in accordance with contemporary trends, and adopts a macro-level perspective of the layout of the leisure sports industry, development strategy and policy design. Yang Yukai (2017), after studying the development of the leisure sports industry in developed countries, concludes that China's leisure sports industry needs to acknowledge the leading role of leisure sports culture in the healthy development of leisure sports, grasp the brand effect and integrate the industrial chain; this, he observes, will promote its own development.

Zhao Lefa (2017) uses the 'diamond model' of strategic management to analyze factors that restrict the competitiveness of China's leisure sports industry. He cites the lag of the mass consumption concept, the small scale of the leisure sports industry, limited integration with other industries and a lack of professional talent. In response, he suggests it is necessary to deepen the health concept, strengthen macro 
guidance, integrate cross-border industry integration and broaden talent training channels. Yang Lei's comparative research finds that, while the market of China's leisure sports industry has great potential, it has not yet shown short-term economic effects. Lei suggests that the sound development of China's leisure sports industry should be based on an understanding of its basic laws of development and distinctive characteristics. Both should be combined with innovation, coordinated development and scientific management.

The research on China's leisure sports industry mainly focuses on quantitative research. The results of qualitative research are limited. It is necessary to change the research paradigm and produce more theoretically in-depth research results.

When studying China's leisure sports, it is important to explore their function and significance from different angles. The past 20 years have only revealed a few research hotspots and frontier changes in China's leisure sports research, and there is still an ongoing need for further research.

\section{METHODS}

\section{A. Study Setting and Data Collection}

The Chinese Social Science Citation Index (CSSCI) database was selected and documents from the period 1998-2017 were searched by using the keywords "leisure sports" and "leisure". After screening, 371 documents that satisfied the requirements were selected as this study's dataset.

\section{B. Quantitative analysis}

This study uses Visual analysis tools and CiteSpace 5.0 to study research hotspots and leisure sports trends in China that have emerged since the reform and 'opening up'. Their application will avoid subjective judgement and will better demonstrate the process and distribution of leisure sports research. The Citespace 5.0 is installed and run on JAVA platform; it is then processed, before the document data is downloaded by CSSCI. The year slice is set to one year and the threshold value is set to 50. Keyword, author and dispatch agency are generated in the corresponding maps and are then used to analyze the corresponding high frequency vocabulary, core authors and research institutes.

Bibliographic information statistical analysis tools, including SATI3.0 and Microsoft Office Excel 2007, are applied. In addition, the number of papers, organizations, authors and other information in Citespace 5.0 software are summarized and counted for purposes of further analysis.

C. Qualitative analysis

The high citation-rate literature is cut out from a knowledge map, read in full text and then analyzed through a visual map. The high citation-rate literature cut out in the knowledge map is read in full text, and combined with the visualization map and related papers for analysis. Comment on the context, characteristics and future trends of leisure sports development in China, in order to provide reference for new researchers to discover new research points.

\section{DATA ANALYSIS}

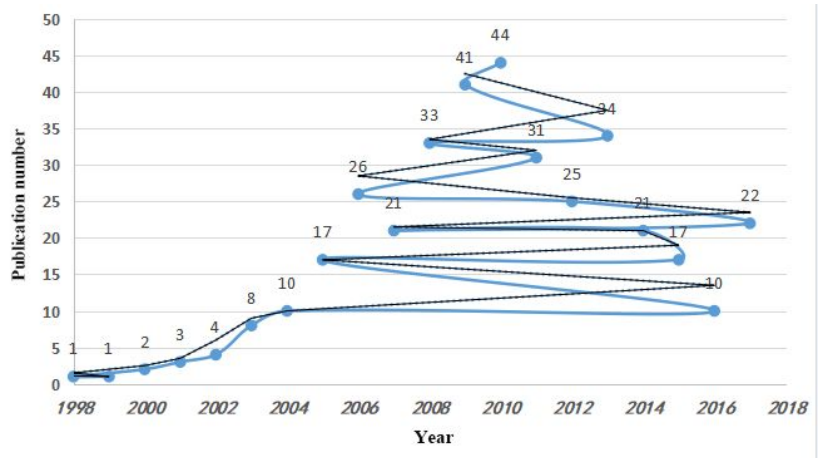

Figure 1. Distribution of leisure sports publications in China: $1998-2017$

The time distribution of knowledge domain research can reflect the overall progress and development of this research field. The number of publications during the period 1998-2017 
(see Fig. 1) show that, from the end of the 1990s onwards, Chinese scholars began to increasingly engage with the study of leisure sports. The concept of 'leisure' was introduced to China for the first time by the scholar Yu Guangyuan, who observes that 'leisure is an important goal of productivity development. The length of leisure time is parallel to the development of humans'.

After it was gradually acknowledged that sports activities were part of leisure activities, research papers on leisure sports began to appear. Since the beginning of the $21 \mathrm{st}$ century, the amount of publications gradually increased, peaking in 2010, and then evidencing a spiral upward distribution trend. Although the volume of papers has declined, the annual number of published papers has remained above 10 .

Changes in the number of articles published on leisure sports were closely related to the development of China's economy and society. The World Leisure Organization observes that per capita GDP of $\$ 2000$ (USD) was the threshold for the rapid growth of leisure demand, and notes that leisure activities began to diversify beyond this point. (Li Xiangru, Ling Ping, Lu Feng,2011) When per capita GDP exceeds \$3000 (USD), leisure demand generally arises. In 2006 and 2008, China's per capita GDP respectively exceeded \$2000 and \$3000 (both USD). Rapid increases in leisure demand led to the development of related research, and reiterated that leisure sports research is a contemporary concern.

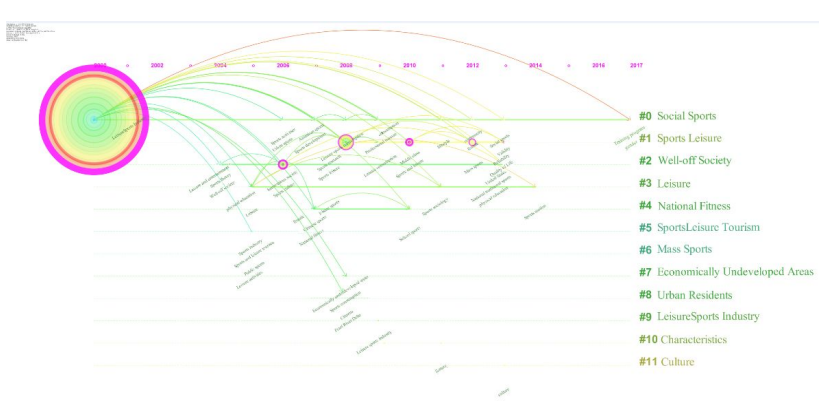

Figure 2. Timeline Analysis of Key Words

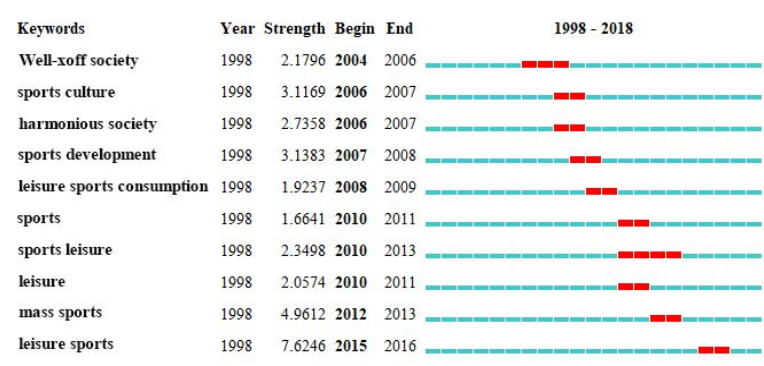

Figure 3. Emergence Information Analysis: Keywords

Leisure sports was a dynamic research field that was closely related to economic and social development in China. Material progress was a precondition for the development of leisure sports, which impacted related research fields. The Time Zone Distribution Chart (Fig. 2) and the keyword chart (Fig. 3) make it possible to identify dynamic changes in research hotspots that have occurred since reform and 'opening up'. The combination of graphic illustrations and interviews with authoritative experts in the field makes it possible to divide research from the last 20 years into three periods:

\section{A. Germination Period (1990s-2000)}

Research of leisure sports in China began in the early 1990s, when scholars began to focus on sports and leisure. Some of the most influential contributions were Leisure Sports Theory (Cheng Zhili, 1990) and Sports and the Leisure Life of Chinese Urban Residents (Liu Depei, 1990). These papers provided theoretical and empirical insight, and highlighted the relationship between social development and the diversification of leisure lifestyle. Due to conceptual underdevelopment, leisure sports was only considered within 'sports and health' and 'social sports', and was even viewed as 'tourism and entertainment', which further underlined its struggle to become an independent discipline. In 1995, China issued an outline of the National Fitness Program, an outline of the Development of the Sports Industry and the Sports Law of the People's Republic of China.

Although research into leisure sports continued to develop, progress was hindered by the under-development of 
concepts and the inability of the field to distinguish itself from its predecessors. This meant that research results for leisure sports remained limited. At this time, China's per capita income was not sufficient to sustain leisure sports, and so related research remained under-developed.

\section{B. Initial period (2001-2005)}

As China became an increasingly developed country in the 21 st century, the research on leisure sports began to progress. Some of the most well-known contributions were: Cultural Significance of the Rise of Leisure Sports (Chen Rong, 2002), Investigation and Development Strategy of Urban Leisure Sports Consumption (Hu Chunwang, 2003), and Sports Leisure Science (Lu Feng, 2005). These contributions considered the significance and value of leisure sports by drawing on cultural, economics, leisure and sports perspectives. At this stage, leisure sports mainly focused on "leisure and entertainment", "mass sports", "well-off society", "entertainment and leisure" and "sports and leisure tourism", in addition to other contents.

\section{Development period (2006-2017)}

During the period 2006-2017, the research hotspots of leisure sports mainly focused on "sports culture", "national fitness", "harmonious society", "American sports development" and "leisure sports consumption". The research results focused on "leisure sports industry" increased substantially. The content involved the basic theory, economic value, educational value, cultural connotation, planning and design of leisure sports. The most influential contributions included Introduction to Leisure

Sports (Xu Zongxiang, 2007), On Sports Leisure ( $\mathrm{Hu}$ Xiaoming, 2008), Thoughts on the Cultivation of Leisure Sports Professionals (Chen Qi, 2008), Thoughts on the Construction of Leisure Sports Specialty in China from the Perspective of Leisure (Li Xiangru, 2009), Formation and Development of Leisure Sports Discipline (Liang Limin, 2010). Introduction to Leisure Sports (Li Xiangru, 2011), Perspective on China's Leisure Sports (Li Xiangru, 2012), Theory and Thinking of China's Leisure Sports Development (Zhong
Bingshu, 2015) and Research on China's Leisure Sports Practice (Li Xiangru, 2016).

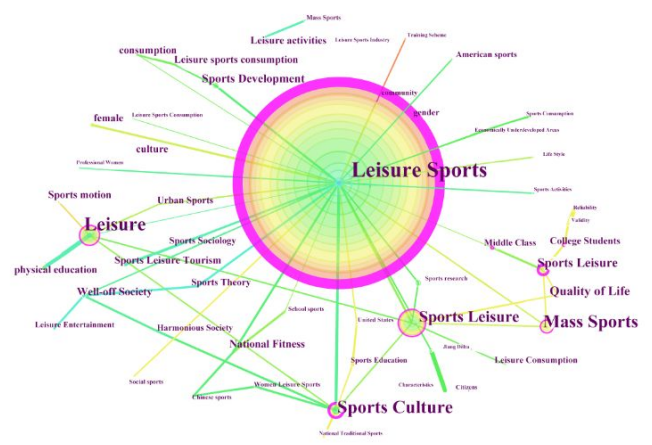

Figure 4. Cluster Co-occurrence Map: Keywords

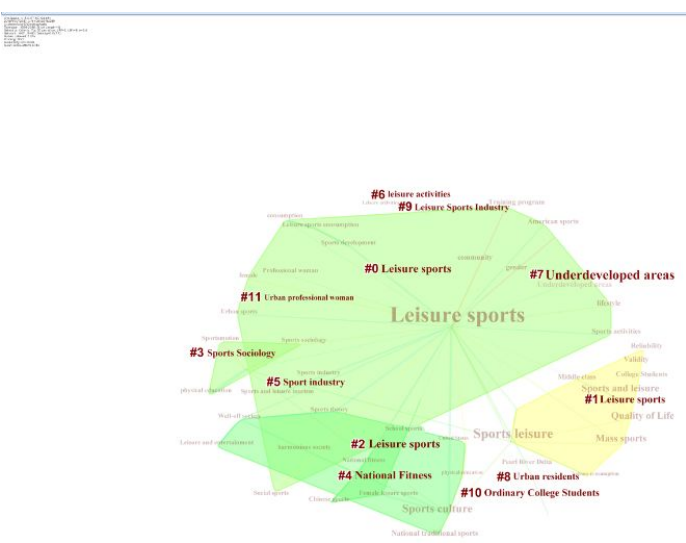

Figure 5. Clustering Common View: Keywords

The keywords in the literature are the core words extracted from the articles. They are core components of an article, and are referenced in the summary and conclusion. Keywords that occur frequency are used to identify hot issues in a research field. The most frequently occurring words collected from CSSCI data are 'leisure sports', 'sports leisure', 'leisure', 'sports culture' and 'sports leisure' (Fig. 4). This shows that, since the reform and 'opening up', scholars in the field of leisure sports have mainly focused on these contents, with the consequence that the research scope of leisure sports in China has generally progressed. Foreign experience, meanwhile, helped to stimulate domestic demand, increase 
employment and improve national happiness. But extended research into leisure sports in China was still insufficient. There were too many theoretical macro-studies and too few empirical micro-papers. Some leisure sports research directly copied from foreign research, and the results were divorced from China's national conditions.

Leisure sports was a compound subject produced by the combination of 'Leisure Science' and 'Sports Science'. The current tendency to integrate disciplines made it clear that leisure sports cannot exist as an isolated island, and therefore needs to be combined with different disciplines to produce new academic breakthroughs. The research scope of leisure sports is currently mainly combined with the contents of 'sports industry', 'national fitness' and 'gender' (Fig. 5). However, there are too many quantitative studies, too few qualitative studies, insufficient research paradigms, no significant interdisciplinary research and insufficient integration with sociology, pedagogy, economics, anthropology and other disciplines.

For example, an exploration of the social significance of leisure sports can draw on sociology, while the behavioral motivations of leisure sports participants can be engaged from the perspective of Social Action Theory. The symbolic meaning of leisure sports can be explored from the perspective of Symbolic Interaction Theory, and the interactive and communicative value of leisure sports can be explored from the perspective of Daily Life Theory. Leisure sports can also be analyzed from the perspective of pedagogy. The interactive mode of leisure sports can be constructed with the theory of experiential learning, and the relationship between leisure sports and human status acquisition and identity can be analyzed by drawing on the theory of educational stratification and conflict. Leisure sports can also be analyzed by drawing on the theory of economics, while the economic value of leisure sports can be explored through theories of human capital and social reproduction. Anthropological perspectives can be drawn on to assess the cultural significance of leisure sports.

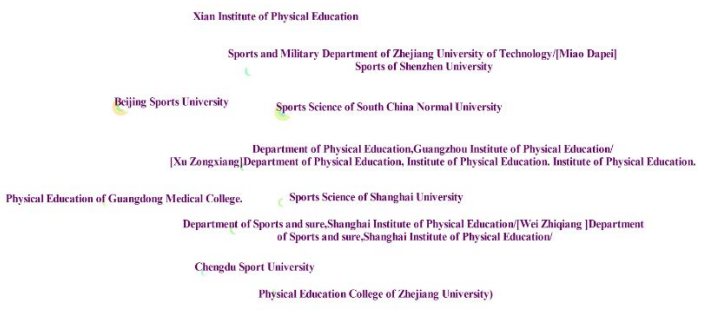

Figure 6. Co-occurrence Map: Research Institutions

Leisure sports have emerged as a 'hot spot' of sports research in recent years. Analysis of the common view spectrum of leisure sports research institutions can provide insight into the core institutions that promoted leisure sports research, and this can help researchers to better understand related research fields from the perspective of research institutions.

Leisure sports research in China is concentrated in different universities (Fig. 6), of which sports and comprehensive universities account for the highest proportion (five and three respectively). Sports colleges and universities tend to focus more on leisure sports research. Comprehensive colleges and universities, meanwhile, engage on an interdisciplinary basis, adopt diverse research perspectives and have a clear advantage with regard to the quality of research. China's leisure sports research institutions tend to be based in economically developed cities, and this reflects both economic thresholds and the objective law of disciplinary development.

While leisure sports research in China has given rise to different views, the total number of views has not been high; furthermore, the nodes of institutions have been relatively isolated, with no connection between them. Leisure sports research institutions have fewer cross-regional alliances, low efficiency of resource integration and greater disciplinary limitations, none of which are conducive to cross-disciplinary integration of leisure sports research. Research institutions should accordingly strengthen cooperation with the intention of 
achieving complementary research resources and should work to promote the development of leisure sports research.

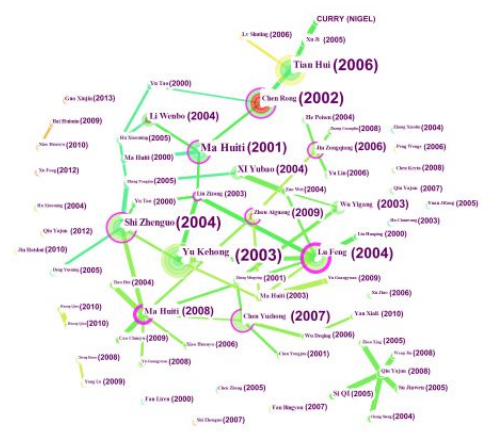

Figure 7. Co-occurrence map: highly-cited literature

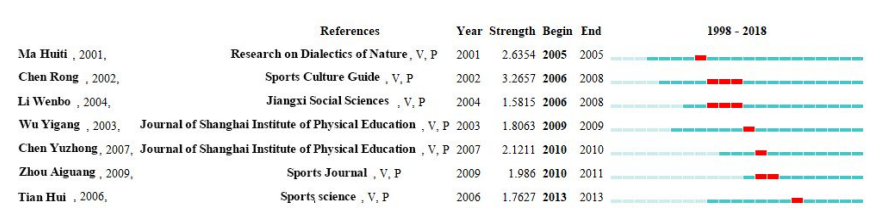

Figure 8. Co-occurrence information analysis

Frequently cited contributions to the literature can re veal the knowledge base of leisure sports research (Fig. 7). The most frequently cited paper is Yu Kehong's 'On Leisure Sports from the Definition of Leisure', which wa s published in China Sports Science and Technology in 2 003. It mainly compares and summarizes the definitions o f 'leisure' and 'leisure sports' put forward by Chinese an $\mathrm{d}$ foreign scholars, with the intention of developing a mo re objective, comprehensive and clear definition. The seco nd paper is Tian Hui's 'Leisure, Leisure Sports and Its D evelopment Trend', which was published in Sports Scienc $e$ in 2006. It mainly analyzes and interprets the meaning of leisure and leisure sports by tracing the historical deve lopment of leisure sports and discusses its content. The $t$ hird is 'Cultural Significance of the Rise of Leisure Sport s' by Chen Rong, which was published in 2002 in Sport

$s$ Culture Guide. This paper adopts a cultural perspective to discuss the cultural characteristics and significance of leisure sports. Shi Zhenguo published 'Leisure, Leisure a nd Leisure Sports' in Sports Culture Guide in 2004. It a nalyzes the historical origins of leisure and leisure sports, and also engages the concept of leisure sports activities. In 2004, Lu Feng's 'Discrimination of Leisure Sports Conc epts' was published in the Journal of Chengdu Institute o $f$ Physical Education. It defines the concept of leisure sp orts and incorporates the author's personal opinions to est ablish three constructive dimensions of leisure sports.

Forward-looking research has also received extensive attention in the research community (Fig. 8). Ma Huidi's '21st Century and Leisure Economy, Leisure Industry and Leisure Culture' was published in Dialectics of Nature in 2001. It anticipates a stronger interrelation of the leisure industry and China's culture and economy during the 21 st century, and also calls for the strengthening of relevant academic research. It was widely cited in 2005. Li Wenbo's 'Leisure Sports Consumption Research: An Interpretation of Culture and Sociology', which was published in Jiangxi Social Sciences in 2004, proposes that sports culture has a unique cultural symbolic significance. It was widely cited during the period 2006-2008.

Wu Yigang's 'Current Situation and Problems of Leisure Sports Research at Home and Abroa" was published in Journal of Shanghai Institute of Physical Education in 2003. It studies changes within leisure sports and their general development of leisure sports at both the domestic and international level, and also acknowledges limiting factors in each of these respects. It was quoted extensively in 2009. Tian Hui's 'Leisure, Leisure Sports and Its Development Trend in China' was published in Sports Science in 2006. It traces the origin and evolution of leisure sports in China and also explains their significance and content. It was widely cited in 2013. Chen Yuzhong's 'Future Trend of Leisure Sports Development in China' was published in Journal of Shanghai Institute of Physical Education in 2007. It explores the conditions and historical stages of the rise of leisure sports in China, and 
provides insight into their future prospects. It was widely cited in 2010. These frontier contributions have made a vital contribution by establishing a basis for the future development of leisure sports research.

\section{Conclusions and Future Research}

\section{A. Conclusions}

The scientific knowledge map of core journal papers on leisure sports from the past 20 years is subject to analysis by Citespace 5.0 software, and this confirms that research on leisure sports spiraled upwards from the beginning of the $21 \mathrm{st}$ century, before peaking in 2010. Although a decline then followed, a relatively constant annual output was maintained. The research process of leisure sports was hierarchical and closely related to social development and policy dynamics.

Leisure sports research institutions mainly focused on professional sports colleges and comprehensive universities, while financial and economic colleges and normal universities paid less attention to leisure sports. There was less cooperation among scientific research institutions, as effective cooperation mechanisms were still absent. Researchers of leisure sports, including Chen Rong, Yu Kehong, Shi Zhenguo, Lu Feng, Tian Hui, Chen Yuzhong and Zhou Aiguang, contributed to the study of leisure sports by offering different perspectives and methods. But cooperation between different authors was still not sufficient. The hotspots and frontiers of leisure sports research were mainly based on policy, history and culture, and focused on industry, history and comparative sports. This research generally tended to be systematic, pluralistic and innovative.

Although substantial achievements have been made in the research of leisure sports in China, there are still some problems and shortcomings: The connotation of leisure sports research is still not sufficiently deep, and most contributions struggle to extend beyond a relatively superficial concept analysis or assessment of prospects. The results of interdisciplinary research have been insufficient to form horizontal and vertical cooperation mechanisms. In addition, there is also a gap between concrete developments and research. For example, as national fitness has improved, leisure sports items, including yachts, hot balloons, racing cars and RV camping, have also increased; this development has not, however, been reflected in domestic research. Although there have been individual breakthroughs in research paradigms, methods and perspectives, the overall impact has fallen short of what is required.

\section{B. Future Research}

In the future, the research on leisure sports can further improve the insufficiency of this research. The depth of connotation of leisure sports research is insufficient. It can be traced from the meta-research level, correctly define the meaning of leisure sports, and carry out theoretical innovation. Interdisciplinary research on leisure sports Too few results should be led by the government level, so that different disciplines can strengthen cooperation and form a sustainable cooperation mechanism; research on leisure sports should be paid attention to, and many emerging leisure sports should receive more attention, such as: Outdoor sports, yachts, motor homes, etc.; enrich the research paradigm of leisure sports, emphasizing the theoretical depth and practical value of leisure sports research through the perspective of qualitative research

When compared against previous research, this research offers a significant methodological innovation. Its application of visualization software has shown how it is possible to provide an objective representation of the frontiers and hotspots of leisure sports development in China over the last 20 years. Visualization analysis saves a considerable amount of time in accessing the literature, and also makes it possible to extract research hotspots and deficiencies in a certain research field from a large number of papers. In future, it can be both applied to other disciplines and also used to open up new areas of research into leisure sports. 


\section{REFERENCES}

[1] Z. The Central Committee of the Communist Party of China, State Council(2016). Outline of the "Healthy China 2030" Program. http://www.gov.cn/xinwen/2016-10/25/content_5124174.htm.

[2] J. Wang Liwei, Zhang Yongjun, Fan Suxiang(2007). Leisure Sports: A Historical Category of Interactive Economic Development. Journal of Chengdu Institute of Physical Education, 33 (5), 5-8.

[3] M. Yu Guangyuan(2005). On the Generally Leisure Society. Beijing: China Economic Publishing House, 12-16.

[4] M. Li Xiangru, Ling Ping, Lu Feng(2011). Introduction to Leisure Sports. Beijing: Higher Education Press, 11.

[5] Z. China's annual average GDP .https://www.kuaiyilicai.com/stats/gl obal/yearly_per_country/g_gdp_per_capita/chn.html.

[6] J. Lu Feng, Liu Xishan, Wen Xiaoyuan(2006). Classification of Leisure Sports Activities. Journal of Wuhan Institute of Physical Education, 40 (12), 59.

[7] M. Li Xiangru, Ling Ping, Lu Feng(2011). Introduction to Leisure Sports. Beijing: Higher Education Press, 160.

[8] J. Yu Kehong, Liang Ruowen(2003). On Leisure Sports from the Definition of Leisure. China Sports Science and Technology, 39(1), 21-23.
[9] J. Tian Hui, Zhou Hong(2006). Leisure and leisure sports and their development trend in China. Sports Science, 26 (4), 67-70.

[10] J. Chen Rong(2002). Cultural Significance of the Rise of Leisure Sports.Sports Culture Guide, (2), 12-13.

[11] J. Shi Zhenguo, Tian Yupu(2004). Leisure, Leisure Sports. Sports Culture Guide, (8): 45-46.

[12] J. Lu Feng(2004). Discrimination of the Concept of Leisure Sports. Journal of Chengdu Institute of Physical Education, 30 (5), 32-34.

[13] J. Ma Huidi(2001). The 21st Century and Leisure Economy, Leisure Industry and Leisure Culture. Research on Dialectics of Nature, 17 (1), 48-52.

[14] J. Li Wenbo(2004). Research on Leisure Sports Consumption: An Interpretation of Culture and Sociology. Jiangxi Social Science, (9), 154-157.

[15] J. Wu Yigang(2003). Current situation and problems of leisure sports research at home and abroad. Journal of Shanghai Institute of Physical Education, 27 (3), 39-43.

[16] J. Tian Hui, Zhou Hong(2006). Leisure and leisure sports and their development trend in China. Sports Science, 26 (4), 67.

[17] J. Chen Yuzhong(2007). Future Trend of Leisure Sports Development in China. Journal of Shanghai Institute of Physical Education, 31 (1), 9. 